\title{
Neutropenia febril en niños con cáncer
}

\author{
Juan Tordecilla C. ; Myriam Campbell B. ${ }^{\text {; }}$; \\ Pilar Joannon $S .{ }^{1}$
}

\section{Febrile neutropenia in children with cancer}

\begin{abstract}
The prompt initiation of empiric antibsolic therapy is imporiont in children with cancer and neutropentia when they become febrile, because bacterial infeclion is the most frequenl cause of morbidity and mortality among ihem. Initial therapy with cloxacilin, amikacin plus cefoperazone was used in 148 febrile episodes occurring to 84 neutropenic labsolute neurrophil counts less than 500 cells per cubic milimater\} children admitted to the hematology and oncology wards of a public, unlversily associaled, children's hospital al northern merropditan Santiago. Chile, from may 1988 throughout lebruory 1992. Polients were basically affected by acule lymphocylic leukemia in = 38), acute myeloid leukemia in $=23\}$ and other solid umors $i n=23\}$ and were aged 9 months 1014 vears and 9 months. In $47.6 \%$ episodes, fever of unknown arigin was the final diagnosis; pneumonia was identilied in $22.1 \%$ coses; bacteremia or baclerial sepsis were contirmed in $14.1 \%$ episodes and in $16.3 \%$ thore were different kinds of dinically defined infections. Stophylococcus aureus was the mosl frequenlly idenified agenl in bacteremias. The initial trealmenl was succesful in $88.7 \%$ of patients with lever of unknown origin and in $78.3 \%$ of the patients with proven infeclions The initial antibiok schedule was either modilied by shifing to ceflazidime insteod of celaperazone or adding melranidazole when fovourable clinical responses were not observed at the end of 72 hours of treatrent-, or adjusled in accordance to bacterial sensitivity lests as necessary. In eight patients amphoter'cin B was olso used, because they had fever alter seven days of Ireatment, without evidence of a specific localization of their infectious process. All potients were treated for al leasl seven days or more. Lehha'ity rale wos $10.7 \%$ and it wos maily associaled to sepsis, pneumonia and lyphlitis. This antibiolic association seems to be elfective as an inilial opprooct to manogemenl of polentially dangerous bacterial inteclıons in tebrile neutropenic child:en wilh cancer.
\end{abstract}

(Key words: feves, neutropenia, chen oherapy, leukemia, lymphocylic, myeloid, acute.]

Con la quimioterapia oncológica se producen a menudo neutropenias severas y prolongadas, que a su vez guardan una relación cuantitativa directa con la incidencia de complicaciones infecciosas en la evolución de los pacientes con cáncer'. Mientras más severa la neutropenia mayor es la frecuencia de bacteremias e infecciones graves ${ }^{2}$. En alrededor de $70 \%$ de los pacientes neutropénicos febriles se puede comprobar alguna forma de infección bacteriana ${ }^{3}$. Con el transcurso del tiempo se ha observado, además, que los agentes infecciosos encontrados en los nifos neutropénicos cambian. Actualmente se ha hecho más frecuente el aislamiento e identificación de bacterias Gram positivas que Gram negativas ${ }^{4-7}$.

1. Unidad de Hematología-Oncología, Hospital Roberto del Róo.
Un avance significativo en el manejo de pacientes con defectos de la inmunidad ha sido el empleo temprano de diversas combinaciones de antibióticos, tan pronto sufren fiebre. La elección de los antibióticos debe basarse en los antecedentes y manifestaciones clínicas, la magnitud y características locales de los riesgos de infecciones intrahospitalarias y de la comunidad, así como la sensibilidad de los patógenos más importantes, aun cuando el sitio de infección no sea evidente 5.9 . Hace algunos años se mostraron las ventajas del empleo de una combinación de cloxacilina, amikacina y cefoperazona en este tipo de enfermos ${ }^{10}$ y la asociación continúa en uso. El propósito de esta publicación es describir los resultados obtenidos en un periodo de casi cuatro años, siguientes al informe recién aludido, en el manejo de neutropenias febriles. 


\section{Pacientes y método}

Entre los meses de mayo de 1988 y febrero de 1992 , ambos incluidos, sc estudiaron en la Ĺnidad de Hematología y Oncología det Hospital Roberto del Río, 84 niños que, mientras estaban sometidos a quimioterapia oncológica sufrieron uno o más episodios -cada uno- de neutropenia febril. Treinta y ocho de los pacientes estaban afectados por leucemia linfocítica aguds, 23 por leucemia mieloide aguda y otros 23 por tumores sólidos y sus edades cstaban ubicadas entre 9 mescs y 14 años 9 meses. En cinco enfermos se habja ínstalado un caléter venoso central con trayeclo proximal subcutáneo. Los niños con leuccmia estaban recibiendo cotrimoxazol (sulfametoxazol y trimetoprima) oral y colutorios de nustatina, desde la segunda semana de la fase de inducción hasta el inicio de la de mantención, como profilaxis contra infecciones por Pneumocisris carinii y hongos respectivamente. Todos los pacientes con neutropenia (recuento absoluto de neatróflos menos de 500 por $\mathrm{mm}^{3}$ ) y fiebre $\left(\mathrm{T}^{\circ} 38\right.$ en dos registros separados por cuatro horas o una medición de $39^{\circ}$ C) fueron hospilalizados. Además de la historia y el cxamen físico, en todos los episodios se hicieton radiografías de tórax. se procesaron cultivos de sangre, orina, deposición, faringe y de cualquier lesión específica que existiese y se administró una combinación de cloxacilina, amikacina y cefoperazon a ${ }^{10}$. La temperatura se controlo cada cuatro horas y el hemograma cada tres días. De acuerdo con las recomendaciones de un panel internacional de consenso se consideró como buena respuesta clínica la caida de la fiebre dentro de las primeras 72 horas de iniciado el tratamiento". En caso de persistencía de la fiebre más attá de ese tiempo se ioma ron nucvas muestras para cultivos y se cambiaron los antibióticos, empleando cefiazidima en lugar de cefoperazona, agregando metronidazol o adccuando las indicaciones a los resultados de los cultivos iniciales, la sensibilidad de los gêmenes aislados y las manifestaciones clínicas de la infección. Si al séptimo día de evolución el niño seguía con fiebre y nculropcnia y aún no se idenificaba un foco infeccioso específico se agregó anfotericina $\mathrm{B}$ al tratamiento. La duración mínima del tratamicnto fue sicte dias. Los criterios aplicados para suspender los antibióticos fueron: auscncia de fiebre por 48 horas, recuperación del recuento absoluto de neutrófilos a más de 500 por $\mathrm{mm}^{3}$ y evolución clínica favorable.

Los pacientes fueron clasificados er gnipos de diagnóstico de acuerdo a las signientes defjniciones: ficbre de origen desconocido, cuando la evolución clínica y de laboratorio no permitió identíficar un sitio de infección y un agente responsable; bacteremia al desartollo microbiano en uno o dos hemocultivos; sepsis al deterioro clínico progresivo, con hipotensión arterial y signos de falla orgánica, con o sin bacteremia demostrada en los hemoculivos; neumonía y neumonitis a manifestaciones clínicas de afección pulmonar confirmadas por las correspondicntes alteraciones en la radiografía de tórax; tiflitis en presencia de dolor abdominal, vónitos, desplazamiento de asa intestinales, signos de íleo en la radiografia simple de abdomen o de líquido peritoneal libre en la ultrasonografía ${ }^{13}$, infección del tracio urinario y pielonefritis al aislamiento de un germen único en el cultivo de orina y celulitis al eritema indurado, caliente y doloroso del tejido subcutáneo. Las afecciones óticas fueron ratificadas por el otorrinolarngólogo y la amigdalitis fue diagnosticada por la prescncia de exudado sobre las amigdalas faríngeas.

\section{Resultados}

Durante el período de 46 meses, que corresponde a esta descripción, se registraron 149 episodios de neutropenia febril en los 84 niños afectados. La media de la duración de la neutropenia fue 8 días, Ios márgenes 3 y 16 días. En $47 \%$ de los episodios los pacientes tenían menos de 100 neutrófilos por $\mathrm{mm}^{3}$ de sangre. La mayoría de los episodios que ocurrieron cn los pacientes con lcucemias linfocíticas y mieloides agudas (85 a $90 \%$ ) se detectaron en el momento del diagnóstico o en el período de la inducción de la remisión. En los tumores sólidos el episodio se sucedió siempre después de un bloque de quimioterapia (tabla 1).

De acuerdo con las definiciones empleadas, $71(47,6 \%)$ episodios correspondieron a fiebre de origen obscuro; $33(22,1 \%)$ a neumonia o neumonitis; 21 (14,2\%) a bacteremia, fungemia o sepsis y $14(9,4 \%)$ fueron clasificados como diarrea aguda $(n=10)$ y tiflitis $(n=4)$. Se registraron también 4 casos de infecciones urinarias $(2,7 \%)$, dos de otitis y uno de amigdalitis.

Encontramos 18/298 hemocultivos positivos. De ellos, en 10 hubo desarrollo de cocáccas Gram positivas: Staphylococcus aureus $(\mathrm{n}=7)$, Staphylococcus coagulasa negativo $(n=2)$ y Streptococcus sp. $(\mathrm{n}=1)$; bacilos Gram negativos en 5: Klebsiella pneumoniae y Pseudomonas aeruginosa en dos casos cada una y Escherichia coli en el restante; en 3 casos se aisló Candida albicans. En tres de los cinco niños con catéter vcnoso central subcutáneo sc aislaron agentes Staphylococcus aureus, Staphylococcus coagulasa negativo y Candida albicans respectivamente.

\section{Tabla}

Neutropenia febril (149 episodies)

\begin{tabular}{lllr}
\hline Enfermedad basal & n & Etapa tratamiento & \\
\hline Leucemia linfática ag. & \multirow{2}{*}{51} & Diagnóstico & 13 \\
& & Inducción & 28 \\
& & Mantención & 3 \\
Leucernia mieloide & \multirow{2}{*}{45} & Recaída & 7 \\
& & Diagnóstico & 9 \\
& & Inducción & 32 \\
\multirow{2}{*}{ Tumor sólido } & & Mantención & 2 \\
& \multirow{2}{*}{53} & Recaida & 2 \\
& & Postquimioterapia & 53 \\
& & &
\end{tabular}


De los 71 episodios de ficbre de origen oscuro, se obtuvo una buena respuesta en $63(88,7 \%)$, cn ocho casos fue preciso cambiar los antibióticos y en sólo tres se llego al uso de antifúngicos.

En 33 cuadros respiratorios, la respuesta lue buena en $28(84,8 \%)$ de los episodios, en cinco casos fue necesario cambiar de antibiótico y sólo en uno se usó anfotericina B por sospecha fundada de hongos. Los 21 episodios de bacteremia, fungemia o sepsis requirieron un número mayor de cambios de antibiólicos (6/21): en cinco casos se usó ceftazidima por no existir respuesta o por agravamiento del niño y en uno vancomicina por bacteremia a Staphylococcus coagulasa negativo resistente a cloxacilina; en cuatro casos se usó anfotcricina $\mathrm{B}$, en tres de cllos por candidcmia demostrada y en uno por sospecha clínica de sobreinfección por hongos en un episodio de bacteremia a Streptococcus sp. La tiflitis, asociada a leucemia mieloide aguda con menos de 100 neutrófilos por $\mathrm{mm}^{3}$, cvolución clínica arrastrada y grave, fue la principal causa de modificación del tratamiento con antibióticos en los cpisodios de ubicación digestiva: usamos metronidazol cn tres episodios. Los dos casos de pielonefritis ocurrieron a una paciente con rabdiomiosarcoma paravesical y nefrostomía definiliva y uvieron a Pseudomonas aeruginosa como agente causal. Las otras infecciones del tracto urinario fueron causadas por Escherichio coli $y$ Klebsiella pneumoniae respectivamente. En conjunto las infecciones urinarias respondieron bien a la terapia. De las tres celulitis, una fuc de ubicación periorbitaria y en ella no se aisló cl agente causal; otra se localizó en una cxtremidad inferior y una en la región de la sien; estas dos últimas fueron producidas por Psetudonomas aeruginosa, una de ellas resistente a cefoperazona por lo que se cambió de antibiótico a ceftazidima. La otitis y una amigdalitis -esta última por asociación fusocspirilar en el momento del diagnóstico de un paciente con leucemia linfática aguda-, respondieron en buena forma al tratamicnto con antibióticos.

Nueve pacientes de este estudio fallecieron y lodos correspondían a niños cuya infección tenía un foco preciso: siete sufrían leucemia micloide aguda y estaban en una etapa terapćutica de inducción o de reinducción (tres por neumonía, dos por septicemia y dos por tiflitis), una paciente con leucemia linfática aguda fallcció en la ctapa de diagnóstico a causa de septicemia aso- ciada a probable hemorragia intracraneana y un paciente con tumor de Wilms murió, después de la quimioterapia, debido a neumonía extensa.

\section{Comentario}

El análisis de estos resultados sugiere que el plantcamiento terapéutico es adecuado, tomando en cuenta la baja proporción de casos en que debió modificarse el esquema antibiótico. En él destacan, por un lado, la actitud agresiva precoz, diagnóstica y terapéutica y la aplicación de una combinación de antibióticos concordante con la experiencia local sobre la frecuencia de los gérmenes habituales y su sensibilidad ${ }^{13}$.

El hallazgo de ficbre de origen obscuro en la mayoría de los episodios de leucopenia y fiebre concuerda con lo descrito en otras experiencias nacionales ${ }^{14,15}$ y es levemente más baja que en informes de otros países ${ }^{16,17}$ y podría ser explicada por la precocidad con que se aplicó el tratamiento con anlibióticos. Del mismo modo, los niños con fiebre de origen obscuro mostraron la mejor respucsta al conjunto de antibióticos empleados durante el período de neutropenia y no se observó letalidad entre ellos, tal como ha sido descrito habitualmente ${ }^{4,13,18}$. El predominio de bacterias Grampositivas en los cultivos confirma la tendencia observada al cambio en los tipos de agentes responsables de las infecciones documentadas en pacientes neutropénicos, especialmente en lo que respecta a Staphylococcus coagulasa negativo, Staphylococcus aureus y Strepcoccus $s p$. Es posible que cste cambio se deba más bien a modificaciones de la flora intestinal que al uso de catétercs, ya que se registró solamente en dos de cinco pacientes con catéter venoso.

Bactercmia, septicemia y tiflitis fueron los diagnósticos que se asociaron a las mayores modificaciones del esquema de antibióticos propuesto y a la vez fucron las principales causales de muerte cn los pacientes que se comentan. Esto se puede explicar por la gravedad del síndrome clínico, la neutropenia menor de $100 \mathrm{~mm}^{3}$ en la mayoria de estos niños y a la leucemia mieloide aguda que sufrían siete de los nueve casos.

Las infeccioncs por hongos son tambiên una causa importante de morbilidad y morblidad en los niños con defectos de la inmunidad. Los factores predisponcntes incluyen neutropenia, me- 
dicamentos inmunosupresores, catéteres centrales y antibióticos de "amplio espectro". La Candida albicans es el agente causal más común de este tipo de infecciones en los nir̂os con cáncer. La frecuencia con que fue preciso emplear anfotericina $\mathrm{B}$ y con que se comprobó candidemia son similares a lo dcscrito en otras experiencias ${ }^{21}, 22$.

Otros autores incluyen la vancomicina en esquema inicial de antibióticos para el mancjo de pacientes neutropénicos febriles, por la crecientc frecuencia de cepas de Siaphylococcus coagulasa negalivo ${ }^{5,19}$. De acuerdo con esta experiencia y en nuestra realidad local, no sería necesario usarla al comienzo del tratamiento sino más bien reservarla para los casos de cultivos positivos a Staphylococcus resistentes a cloxacilina.

Este estudio subraya la importancia de rcgistrar y analizar las experiencias locales para disehar los protocolos de estudio y tratamiento adccuados.

\section{Resumen}

El comienzo temprano y empírico de diversas combinaciones de antibióticos es obligatorio en niños con cáncer y neutropenia febril, debido a que las infecciones bacterianas son la causa más frecuente de morbilidad y mortalidad entre ellos. En una unidad de hematología y oncología se cstudiaron entre mayo de 1988 y febrero de 1992 , 149 episodios febriles que sucedieron en $84 \mathrm{ni}$ nos con ncutropenia (recuento absoluto de neutrófilos menos de $500 \mathrm{~mm}^{3}$ ). Los pacientes sufrían de lcucemia linfocítica aguda $(n=28)$, leucemia mieloide aguda $(n=23)$ y otros tumores solidos $(n=23)$. Sus edades variaban entre 9 meses y 14 años 9 meses. Como tratamiento inicial se usó la combinación de cloxacilina con amikacina y celoperazona. De los 149 episodios, 51 se observaron en leucemia linfática aguda, 45 en leuccmia mieloide aguda y 53 en tumores sólidos. En $47,6 \%$ de los episodios el diagnóstico final fue fiebre de origen desconocido; en $22,1 \%$ neumonía aguda; $14,1 \%$ fueron considerados bacteremia o sepsis y $16,3 \%$ tenían in lecciones clínicamente definidas (síndrome de diarrea aguda, tiflitis, infección urinaria, pielonefritis, celulitis, otitis y amigdalitis aguda). Las bacterias Gram positivas fucron las encontradas con más frecuencia en los hemocutuvos. El tratamiento inicial fue efectivo en $88,7 \%$ en los pa- cientes con fiebre de origen no precisado y en $78,3 \%$ de los niños con infección definida. La modificación de los antibióticos consistió en el empleo de ceftazimida en lugar de cefoperazona y en la adición de anfotericina $B$ en ocho pacientes por posible sepsis con hongos. Todos los pacientes fucron tratados por siete o más días. La letalidad fue $10,7 \%$ y las principales causas de muerte fueron neumonía, sepsis y tiflitis. La asociación de antibióticos usada parece adecuada para tratar a los pacientes con cáncer que sufren neutropenia febril.

(Palabras clave: fiebre, neutropenia, quimioterapia, antibióticos, leucemia, linfocítica, mieloide, aguda.)

\section{Referencias}

1. Pizzo PA: Evaluation of fever in the patient with cancer Eur J Cancer Clin Oncol 1989; 2 (Suppl 2): 9-12.

2. Bodey GP, Buckey M, Sathe YS, Freireich EJ: Quantitative relationship between circulating leucocytes and infection in patients with acute leukemia. Ann Intem Med 1966; 64: 328-340.

3. Hughes WT: Empiric antimicrobial therapy in the febrile granulucytopenic patient. Infect Controd Hosp Epidemiol 1990: 11: 151-156.

4. Pizzo PA, Hathorn JW, Hiemenz $J$ et al: : A randomized 1rial comparing ceftazidime alone with combindtion antibiotic therapy in cancer patients with fever and neurropenia. $X$ Engl J Med 1986; 315: 552-558.

5. Shenep JL, Hughes WT, Roberson PK el al.: Vancomycin, ticarcillin, and amikacin compared with ticarcillin-clavulanate and amikacin in the empirical treatment of Jebrile, neutropenic children with cancer. $X$ Engl J Med 1988; 319: 1053-1058.

6. Soarinen UM: Severe infections in childhood leukemia. Acta Paediatr Scand 1984; 73: 515-522.

7. Hathorn JW, Pizzo PA: Infectious complications in the pediatric cancer patient. In: Pizzo PA, Poplack DG. Principles and Practice of Pediatric Oncology. Philadelphia, Lippincoll, 1989:837-867.

8. Pizzo PA, Rubin M, Freifeld $A_{1}$ Walsh $T J$ : The child with cancer and infection. Empiric therapy for fever and neutropenia, and preventive stralegies. J Pediatr 1991; 119: 679-694.

9. Hughes WT, Armsirong $D$, Bodey GP el al,: Guidelines for the usc of antimicrobial agents in neutropenic patients unexplained fever. I Infect Dis 1990; 161: 381-396.

10. Joannon $P$, Tordecilla $J$, Campbell $M$ : Síndrome febril en pacientes neutropénicos con cáncer. Consideraciones etiológicas y terapéticas. Pediatría (Santiago) 1988; 31: 231 (Resumen).

11. Pizzo PA, Armstrong $D$, Bodey $G$ at al.: Report of a consensus panel from the Inmunocompromised Host Sociely: The design, analysis, and reporting of clinicals trials on the cmpirical antibjotic management of the neutropenic patienl. J Infecı Dis 1990; 161 : $397-401$. 
12. Bravo M, Tordecilla J, Emparanza E, Campbell $M$. Vildósota $\mathrm{J}$ : Enterocolitis ileocecal aguda (uflitis) en pacientes con neulropenia asociada a quimiotcrapia. Rev Chil Pediatr 1988; 59: 369-373.

13. Riikonen $P$ : Imipenem compared with ceftazidime plus vancomycin as initial therapy fur fever in neutropenic children with cancer. Pediatr Infect Dis J 1991; 10: 918.923 .

14. Salgado C, Becker A, Campbeit M. el al.: Acute lym. phoblastic leukemia: febrile episodes in 60 patients in Chilean National Protocol 1987. Am J Pedjatr Hernatol Oncol 1990; 12: 238 (Abstract).

15. García H, Vargas $L$, Layte C: Neul ropenia previa al uatamiento quimiolerápico en leucemia linfática aguda. Rey Chil Pedialr 1992; 63: 26-31.

16. Granowetren L, Wells $H$, Lange BJ: Ceftazidime with or without vancomycin vs cephalothin, carbenicillin and gentamicin as initial therapy of the febrile ncutropenic pedialt cancer patient. Pediatr Infect Dis J 1988; $7: 165 \cdot 170$.
17. Armstrong D: Empiric therapy for the immunocompromised host. Rev Infect Dis 1991: 13 (Suppl 9): 763-769.

18. Mullen $C A$, Buchaman $G R$ : Early hospital discharge of children with cancer treated for fever and neutropenia: identification and management of the fow risk patient. J Clin Oncol 1990; 8: 1998-2004.

19. Rubin M, Hathorn JW, Marshall D, Gress J, Steinberg SM, Pizzo PA: Gram-Positive infections and use of vancomycin in 550 episodes of fever and neutropenia. Ann Interm Med 1988; 108: 30-35.

20. Wade JC: Management of infection in patients with acute leukemia. Hematol Oncol Clin North Am 1993; 7: 293-315.

21. Pizzo PA, Walsh TJ: Fungal infections in the pediatric cancer patient. Semin Oncol 1990; 17 (Suppl 6): 6-9.

22. Willey $J M$, Smilh $N$, Leverhal $B G$ et al.: Invasive fungal disease in pediatric acute leukemia patients with fever and neutropenia during induction chemotherapy: a multivariate analysis of risk factors. J Clin Oncol 1990; 8: $280-286$.

\section{AVISO A LOS AUTORES}

Con el objeto de acelerar la edición de los trabajos se solicita encarecidamente a los autores que disponen de computador con procesadores de texto IBM compatibles, enviar - en lo posible junto con las dos copias impresas de su material-, otra en disco flexible (tamaño 3,5" o 5,25", de doble o alta densidad) del mismo texto. El disco será devuelto de inmediato por correo certificado o por mano al autor corresponsal. 